\title{
Acquiring Paraphrases from Text Corpora
}

\author{
Rahul Bhagat \\ USC Information Sciences \\ Institute \\ Marina del Rey, CA \\ rahul@isi.edu
}

\author{
Eduard Hovy \\ USC Information Sciences \\ Institute \\ Marina del Rey, CA \\ hovy@isi.edu
}

\author{
Siddharth Patwardhan \\ School of Computing \\ University of Utah \\ Salt Lake City, UT \\ sidd@cs.utah.edu
}

\begin{abstract}
Paraphrases are textual expressions that convey the same meaning using different surface forms. Capturing the variability of language, they play an important role in many natural language applications including question answering, machine translation, and multidocument summarization. In linguistics, paraphrases are characterized by approximate conceptual equivalence. Since no automated semantic interpretation systems available today can identify conceptual equivalence, paraphrases are difficult to acquire without human effort. In this paper, we present a method for automatically acquiring paraphrases using a monolingual corpus. We learn paraphrases at both the surface and lexico-syntactic levels and build two paraphrase resources each containing about 2 million phrases. We evaluate these paraphrases extrinsically by using them to learn patterns for Information Extraction (IE). We show that the lexico-syntactic paraphrases performs better than the surface-level paraphrases for IE. We further show that the patterns learned using the lexicosyntactic paraphrases attain comparable performance to the traditional IE approach of learning patterns from domain-specific corpora.
\end{abstract}

\section{Categories and Subject Descriptors}

I.2.6 [Artificial Intelligence]: Learning-Knowledge acquisition; I.2.7 [Artificial Intelligence]: Natural Language Processing - Text analysis

\section{General Terms}

Algorithms

\section{Keywords}

Paraphrase acquisition, Patterns, Information Extraction

Permission to make digital or hard copies of all or part of this work for personal or classroom use is granted without fee provided that copies are not made or distributed for profit or commercial advantage and that copies bear this notice and the full citation on the first page. To copy otherwise, or republish, to post on servers or to redistribute to lists, requires prior specific permission and/or a fee.

$K$-CAP'09, September 1-4, 2009, Redondo Beach, California, USA.

Copyright 2009 ACM 978-1-60558-658-8/09/09 ...\$10.00.

\section{INTRODUCTION}

Variability is a common phenomenon in language: The meaning conveyed by a sentence or phrase can be expressed in several different ways. For example, the pairs of sentences (1) and (2):

The shuttle Discovery landed in Florida on Saturday.

The shuttle Discovery touched down in Florida on Saturday. (2) or the phrases (3) and (4):
$X$ landed
(3)
$X$ touched down

respectively express the same meaning. Such semantically equivalent sentences and phrases are called paraphrases. The ability to paraphrase gives language the flexibility of articulation. It is hard to imagine a language without this facility: it will become monotonous.

Since paraphrasing is such a common phenomenon in language, every Natural Language Processing (NLP) application has to deal with paraphrases. But, automatically capturing the semantic phenomenon of "approximate conceptual equivalence", that defines paraphrases is hard. However, some people have attempted to capture and harness the power of paraphrases to improve NLP applications and have shown promising results for question answering [14], statistical machine translation [5], and multi-document summarization [2].

In this paper, we present methods to acquire a large number of paraphrases. Using a large monolingual corpus, we focus on acquiring paraphrases with one argument slot (ex: $X$ landed) and learn paraphrases at both the surface and deeper levels. We then use these paraphrases to learn patterns for performing Information Extraction (IE) in various domains. This IE task acts as an extrinsic evaluation for measuring the quality of the learned paraphrases.

The paraphrase based approach for learning IE patterns presented in this paper is different from the traditional approach to IE. The traditional approach is:

1. Obtain a domain-specific corpus.

2. For each type of entity or event-role to be extracted, build or learn the patterns from this corpus.

In contrast, the approach presented in this paper is: 
1. Acquire a large set of paraphrases (of patterns) in advance.

2. For each type of entity or event-role to be extracted, look up patterns from this set of paraphrases.

We compare our approach to various state-of-the-art IE systems trained using the traditional approach. We empirically show that our approach has comparable performance to the traditional approach, despite that fact that our system is not tailored to any specific domain. This makes the task of performing IE on new domains easy and proves that the learned paraphrases are a high quality resource for obtaining state-of-the-art performance for IE.

\section{RELATED WORK}

\subsection{Paraphrases for Pattern Learning}

Paraphrases have emerged as a useful tool for learning IE patterns, especially in the context of open-domain IE $[19,1,3]$. Given that the focus of most of this work is on relation extraction, the majority of the existing paraphrase learning techniques $[11,23,3]$ are tailored to learning paraphrases with two arguments (ex: $X$ went off in $Y$ ). But, when only one-argument is to be extracted, simply applying these two-argument techniques leads to very low recall. Our focus in this paper is on the one-argument case, hence our method learns paraphrases with a single argument (ex: $X$ went off ). Paraphrase learning in our case is different and arguably harder, because two arguments provide stronger constraints on the contexts they match and on the pattern boundary. Incorrect boundary detection in our case means that our patterns will be either too general or too specific. Also, since there are many more potential phrases with one argument, compared to the ones with two, scaling the learning algorithm is harder in our case.

There has however been some past work on learning paraphrases with one argument. [20] used comparable news articles to learn paraphrases. [13] used the discourse structure in text to learn entailing verb templates. Most recently [22] used distributional similarity between paths in dependency trees to learn entailing syntactic templates. All these approaches learn syntactic paraphrases or entailments which involves parsing the text with a dependency parser and learning similar paths. Our first method however learns surface-level paraphrases making it easily scalable to large corpora and giving us the flexibility to apply different levels of post-processing. Our second method learns lexico-syntactic paraphrases, using shallow parsing, which is also several times faster than full parsing. To the best of our knowledge, no one has previously learned paraphrases the way we do, in this paper.

\subsection{Patterns for Information Extraction}

While the pattern based approach to information extraction has been popular since the early 1990's, the initial work focused on manual creation of patterns [10]. Focus however quickly shifted to learning patterns automatically from domain-specific corpora. One line of work focused on using annotated training corpora to learn these patterns $[16,8]$. However, given the need for large amounts of tedious manual annotation for this, weakly-supervised approaches, which need very little annotation, are now becoming popular $[17,12]$. These and other similar approaches to domain-specific IE use domain-specific corpora for learning patterns. Their dependence on domain-specific corpora is a hindrance to the easy portability of these methods to new domains. The pattern-learning method we present here, however, differs from the previous approaches in that it does not need a domain-specific corpus for learning patterns: It learns patterns from a general broad-coverage corpus. Also, our method collects patterns from a broadcoverage corpus only once. Patterns can then be generated for any (new) domain by using just a few seed patterns.

\section{PARAPHRASE ACQUISITION}

The intuition that words (or phrases) that occur in similar contexts should have similar meanings is nicely captured by Harris's Distributional Hypothesis [9]. For example, let's assume that the context (C) of a phrase is a one word window to its left and a one word window to its right. Using this definition of the context and looking at a corpus, one might find that the phrase "went off" has its context $C_{\text {went off }}=\{-1$ :bomb, -1 :carbomb, -1:alarm, +1:in, +1:near, +1 :at, ... $\}$. Another phrase, "exploded" might have its context $C_{\text {exploded }}$ $=\{-1$ :bomb,-1 :car-bomb,-1 :cylinder,+1 :in, +1 :on, +1 :at, ... $\}$. Since contexts of these two phrases are similar, it is assumed (correctly) by distributional hypothesis that the phrases "went off" and "exploded" are similar. In this paper, we use this intuition of distributional similarity to learn paraphrases for surface-level and lexico-syntactic patterns that contain one variableslot (argument).

\subsection{Acquisition of Surface-level Paraphrases}

Let $p$ be a pattern of the form "〈SLOT $\rangle$-gram" or "n-gram $\langle\mathrm{SLOT}\rangle$ ", where "〈SLOT $\rangle "$ contains a word or phrase that we expect $p$ to extract, and " $n$-gram" is any n-gram in a corpus. Let's call the word or phrase that $p$ extracts its slot-filler. The aim of the paraphrase learning model is to find a set of patterns that are similar in meaning to $p$.

Let $P=\left\{p_{1}, p_{2}, p_{3}, \ldots, p_{l}\right\}$ be the set of all patterns of the form " $\langle\mathrm{SLOT}\rangle$ n-gram" or " $n$-gram $\langle\mathrm{SLOT}\rangle$ ". Let's define the context $C_{i}$ of a pattern $p_{i} \in P$ to be - its slot-filler and a two word (token) window on its other 
side. For example, let's assume that we have the following sentence in our corpus:

The bomb went off prematurely.

Let's also assume that we have a pattern " $\langle$ SLOT $\rangle$ went off" in our set of patterns $P$. Given sentence (5) and $p_{i}=$ "〈SLOT $\rangle$ went off", its context is:

$C_{i}=\{\langle\mathrm{SLOT}\rangle:$ bomb, +1 :prematurely, $+2:$. $\}$

Similarly, if we assume the we have the pattern $p_{j}=$ "the $\langle$ SLOT $\rangle$ " in $P$, its context is:

$$
C_{j}=\left\{\langle\mathrm{SLOT}\rangle \text { :bomb, }-1: \mathrm{NULL}^{1},-2: \mathrm{NULL}\right\}
$$

Each item that occurs in the context $C_{i}$ of $p_{i}$, is called a feature of $p_{i}$. For each feature $f \in C_{i}$, we calculate its strength of association with the pattern $p_{i}$ using pointwise mutual information (PMI) [7]. We then construct the feature vector $V_{i}$ associated with $p_{i}$, such that it contains each feature with its associated PMI value. For example, given sentence (5) and the pattern $p_{i}=$ "〈SLOT $\rangle$ went off", its feature vector would be:

$V_{i}=\{\langle\mathrm{SLOT}\rangle$ :bomb 3.53, +1:prematurely $1.74,+2: .2 .76\}$

Once we have feature vectors for all patterns in our corpus, we find paraphrases for each by finding its nearest neighbors using cosine similarity ${ }^{2}$. To make this computation scalable, we use locality sensitive hashing (LSH) [6], which reduces the computational cost by building small signatures for each feature vector. [15] have shown that LSH along with a fast similarity computation algorithm [6] makes the nearest neighbors computation approximately linear in the number of vectors $(n)$ and the dimensionality of the vector space $(d)$, i.e., $O(n d)$.

\subsection{Acquisition of Lexico-syntactic Para- phrases}

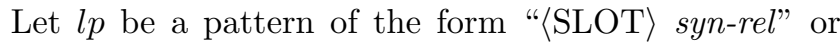
"syn-rel $\langle\mathrm{SLOT}\rangle$ ", where " $\langle\mathrm{SLOT}\rangle$ " contains a word or phrase that we expect $l p$ to extract (slot-filler), and "syn-rel" is a syntactic relation discovered by a shallow parser, in a corpus. Let $L P=\left\{l p_{1}, l p_{2}, l p_{3}, \ldots, l p_{l}\right\}$ be the set of all patterns of the form " $\langle\mathrm{SLOT}\rangle$ synrel" or "syn-rel $\langle\mathrm{SLOT}\rangle$ ". Let's define the context $C_{i}$ of a pattern $l p_{i} \in L P$ to be - its slot-filler. For example, given the sentence (5) in section 4.1 and $l p_{i}=$ "Subject $(\langle$ SLOT $\rangle)$ ActiveVP(exploded)", its context is: $C_{i}=\{\langle\mathrm{SLOT}\rangle:$ bomb $\}$

Using this definition of patterns and their contexts, feature vectors are constructed for all of the lexicosyntactic patterns in a corpus, similar to section 3.1. Using these feature vectors, the paraphrases for each lexico-syntactic pattern are then learned as in section 3.1 .

\footnotetext{
${ }^{1}$ NULL is a dummy feature that indicates that no word was found in the given context.

${ }^{2}$ Cosine similarity between two vectors is the cosine of the angle between them.
}

\section{PATTERNS FOR INFORMATION EX- TRACTION}

In this section, we present our method to learn IE patterns using paraphrases.

\subsection{Learning Surface-Level Paraphrase Pat- terns}

Here, we present our method to learn surface-level paraphrase patterns. Formally, given an event-role $e$ in a domain $d$, the aim is to learn a set of surface-level extraction patterns $S U R F=\left\{\operatorname{surf}_{1}, \operatorname{surf}_{2}, \ldots, \operatorname{surf}_{n}\right\}$ that can extract instances of $e$. For example, given the event role $e=$ "weapon" in the domain $d=$ "terrorism", aim is to learn the following patterns:

$S U R F=\{\langle\mathrm{SLOT}\rangle$ that exploded, $\langle\mathrm{SLOT}\rangle$ blew up, was hit by $\langle\mathrm{SLOT}\rangle, \ldots\}$.

Let's assume that we have a set of surface-level seed patterns $S E E D=\left\{\right.$ seed $_{1}$, seed $_{2}, \ldots$, seed $\left._{m}\right\}$ for the event role $e$. Given $S E E D$, our model finds the paraphrase set $P A R A_{i}$ for each seed $_{i} \in S E E D$. The set of surface-level extraction patterns $S U R F$ for $e$ then is the union of the individual paraphrase sets, i.e., $S U R F=$ $P A R A_{1} \cup P A R A_{2} \cup \ldots \cup P A R A_{m}$. Each pattern in $S U R F$ comes with an associated score, which is the similarity between the learned pattern $\operatorname{surf}_{i} \in S U R F$ and the seed pattern seed $_{j} \in S E E D$ that generated it ${ }^{3}$.

For example, given the event role $e=$ "weapon" in the domain $d=$ "terrorism" and the seeds:

$S E E D=\{\langle\mathrm{SLOT}\rangle$ went off, $\langle\mathrm{SLOT}\rangle$ exploded, ... $\}$

we might find a set of surface patterns, as in (6).

This gives us our surface-level paraphrase extraction patterns - SurfPara.

\subsection{Learning Lexico-Syntactic Paraphrase Patterns by Conversion}

The method described in section 4.1 generates surfacelevel patterns from a set of seed extraction patterns. These patterns are lexical in nature. They extract information by matching the exact sequence of words (tokens) in the pattern with that in the given text and extracting the noun phrase that acts as a slot-filler for that pattern (ex: 〈SLOT〉 exploded). The lexical nature of these patterns makes them very specific. While this specificity often results in high precision extractions, it can be a serious disadvantage, especially when recall is important. For example, we need several variations of surface-level patterns to match all the active voice verb phrases containing the verb " $e x$ -

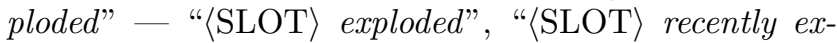
ploded", "〈SLOT $\rangle$ suddenly exploded", etc. However a single lexico-syntactic pattern "Subject( $\langle\mathrm{SLOT}\rangle) A c$ -

\footnotetext{
${ }^{3}$ If a pattern is generated by two or more seed patterns, its score is the average of all the scores it gets from the different seeds.
} 
tiveVP(exploded)" matches them all. Since, extracting specific entities in a small domain-specific corpus is likely to require high recall, we automatically generalize the surface-level extraction patterns.

Formally, let $S U R F=\left\{\operatorname{surf}_{1}, \operatorname{surf}_{2}, \ldots, \operatorname{surf}_{n}\right\}$ be a set of surface-level extraction patterns. The aim is to convert them into a corresponding set of lexico-syntactic extraction patterns $L E X S Y N=\left\{\right.$ lexsyn $_{1}$, lexsyn $_{2}, \ldots$, lexsyn $\left._{m}\right\}$.

To convert the surface-level patterns into lexicosyntactic patterns, we use a shallow parser. We parse all the surface patterns $\operatorname{surf}_{i} \in S U R F$ using this shallow parser and generalize them based on certain lexical and syntactic dimensions:

Voice: The different lexical variations of the active and the passive voice forms of a verb all map to the corresponding general active and passive representations. For example, "〈SLOT $\rangle$ exploded", "〈SLOT $\rangle$ recently exploded", "〈SLOT $\rangle$ suddenly exploded" all contain active voice forms of the verb "exploded", and hence map to the lexico-syntactic pattern "Subject $(\langle\mathrm{SLOT}\rangle) \mathrm{Ac}$ tiveVP(exploded)".

Head word: All noun phrases, verb phrases, prepositional phrases, and adjectival phrases in the surface pattern are represented only by their respective heads. For example, given the pattern "the recent explosion of $\langle\mathrm{SLOT}\rangle$ ", it is generalized to the form "NP(explosion) of $\langle\mathrm{SLOT}\rangle$ ", which is considered equivalent to "the loud explosion of $\langle\mathrm{SLOT}\rangle "$ ", "the explosion of $\langle\mathrm{SLOT}\rangle "$.

Syntactic templates: We use a pre-defined set of syntactic templates to add only those patterns that match these templates into our set of lexico-syntactic patterns $L E X S Y N$. For example, "SSubject〉 ActiveVP" is a syntactic template. Using this template, the surfacelevel pattern "〈SLOT $\rangle$ recently exploded" results in the pattern "Subject( $\langle\mathrm{SLOT}\rangle) \operatorname{ActiveVP}($ exploded $) " 4$, which would be added to LEXSYN.

After performing these generalizations, we get our first set of lexico-syntactic paraphrase extraction patterns LexSynPara (Conv).

\subsection{Learning Lexico-Syntactic Paraphrase Patterns Directly}

Here, we present our method to learn lexico-syntactic paraphrase patterns from a corpus. Formally, given an event-role $e$ in a domain $d$, the aim is to learn a set of lexico-syntactic patterns $L E X S Y N=\left\{\right.$ lexsyn $_{1}$, lexsyn $_{2}, \ldots$, lexsyn $\left._{n}\right\}$ that can extract instances of $e$. For example, given the event role $e=$ "weapon" in the domain $d=$ "terrorism", the aim is to learn the following patterns:

\footnotetext{
${ }^{4}$ If multiple templates match a given surface-level pattern, then a lexico-syntactic pattern is generated for each matched construct.
}

LEXSYN = \{ActiveVP $($ detonated $)$ DirObject $(\langle\mathrm{SLOT}\rangle)$, ActiveInfVP (wants defuse $)$ DirObject $(\langle\mathrm{SLOT}\rangle), \ldots\}$.

The set of lexico-syntactic extraction patterns $L E X S Y N$, for an event-role $e$ in a domain $d$, is then constructed using a set of lexico-syntactic seed patterns $L S E E D$, similar to section 4.1.

For example, given the event role $e=$ "weapon" in the domain $d=$ "terrorism" and the set of seeds:

$L S E E D=\{$ Subject $(\langle\mathrm{SLOT}\rangle) \operatorname{ActiveVP}($ exploded $)$, Subject $(\langle\mathrm{SLOT}\rangle)$ PassiveVP $($ defused $), \ldots\}$

we might find a set of lexico-syntactic patterns, as in (7).

This gives us our second set of lexico-syntactic paraphrase patterns - LexSynPara (Direct).

\section{EXPERIMENTAL METHODOLOGY}

In this section, we summarize the experiments and results of learning the surface-level and lexico-syntactic paraphrases and using them to learn extraction patterns for various event-roles.

\subsection{Paraphrases and IE Patterns}

For learning the broad coverage paraphrases, we used a 2.2 billion word corpus consisting of mainly newswire data. It contains data from the English gigaword corpus which has about 1.75 billion tokens collected from various international new sources; the HARD 2004 text corpus, consisting of about 225 million words of newswire and web text; and the CSR-III text corpus, consisting of about 225 million words of newswire text.

For learning surface-level paraphrases, we part-ofspeech (POS) tagged the corpus using the Brill POS tagger [4] and applied the method described in Section 3.1 to the corpus. We assume that every noun (or a sequence of nouns) that occurs in our corpus is a potential slot-filler. We enumerate every $\mathrm{n}$-gram around that noun (or sequence of nouns), upto a maximum size of three as a candidate pattern for paraphrase learning. We discard all patterns that occur less than 100 times in the corpus (for scalability). We eventually build a surface-level paraphrase resource containing over 2 million patterns, where each pattern is connected to its paraphrases $^{5}$. We then create 10 seeds for each eventrole we want to extract. We then look up paraphrases for each of these seed patterns from the surface-level paraphrase resource and obtain surface-level patterns, as described in Section 4.1.

Once we have the surface-level paraphrase patterns, we convert them into lexico-syntactic patterns as described in section 4.2. To perform this conversion, we use the pattern generation component of the AutoSlog

\footnotetext{
${ }^{5}$ A similarity threshold of 0.1 is set for the paraphrases, based on data inspection and prior experience.
} 
system [16]. We use the Sundance shallow parser [18] and the default 17 pattern templates that are a part of this system package (e.g., 〈Subject〉 PassiveVP, ActiveVP $\langle$ DirObject $\rangle$, etc.).

Finally, for learning the lexico-syntactic paraphrases, we applied the method described in Section 3.2 to the corpus. We used the Sundance shallow parser [18], the default 17 pattern templates that are a part of this system package (e.g., 〈Subject〉 PassiveVP, ActiveVP (DirObject $\rangle$, etc.), and the pattern generation component of the AutoSlog system [16], to generate every pattern occurring in the corpus. We assume that every noun phrase that matches a pattern in our corpus is a potential slot-filler. We discard all patterns that occur less than 10 times in the corpus (for scalability). We eventually build a lexico-syntactic paraphrase resource containing over 2 million patterns, where each pattern is connected to its paraphrases ${ }^{6}$. We then convert the 10 surface-level seed patterns (above) for each event-role into lexico-syntactic forms ${ }^{7}$. We then look up paraphrases for each of these lexico-syntactic seed patterns from the lexico-syntactic paraphrase resource and learn the lexico-syntactic paraphrase patterns, as described in Section 4.3

\subsection{Test Data}

To test our IE systems, we used data from three standard IE data-sets belonging to three domains: terrorism, disease-outbreaks, and corporate-acquisitions.

For the terrorism domain, we used the MUC-4 terrorism corpus [21], which consists of Latin American terrorist events. It has a total of 400 gold-standard annotated documents in its test portion, divided into four sets of 100 each (TST1, TST2, TST3, and TST4). Of these, the TST1 and TST2 documents were used for tuning, TST3 and TST4 documents were used for test. We focused on extracting five event roles in this domain: perpetrator individuals, perpetrator organizations, physical targets, victims, and weapons.

For the disease-outbreaks domain, we used a ProMedmail ${ }^{8}$ IE data set, which consists of reports about outbreaks of infectious diseases. This collection consists of 245 gold-standard annotated articles. Of these, 125 were used for tuning and 120 for test. We extracted two event roles in this domain: diseases and victims.

For the corporate-acquisitions domain, we used the corporate-acquisitions corpus [8]. It consists of 600 newspaper articles about acquisitions and mergers of

\footnotetext{
${ }^{6}$ A similarity threshold of 0.1 is set for the paraphrases, based on data inspection and prior experience.

${ }^{7}$ The conversion of 10 surface seeds results in 8-10 lexicosyntactic seeds for each event role, because some surface seeds map to the same lexico-syntactic seed.

${ }^{8}$ http://www.promedmail.org
}

companies. These articles have gold-standard annotations. Of these, we randomly set aside 300 documents for tuning and used the remaining 300 for test. We extracted two event roles in this domain: acquired and purchaser.

\subsection{Evaluation}

The complete event-oriented IE task involves the generation of event templates. Template generation is complicated: It requires discourse analysis to identify the different events in one article and coreference resolution to find coreferring entities. Our aim here, however, is to evaluate the quality of extraction patterns. Hence, following [12], we evaluate our methods on the quality of their extractions rather than on template generation ${ }^{9}$. Also, following [12], we merge duplicate extractions and employ a head noun matching based evaluation scheme: An extraction is correct if its head noun matches the head noun of the gold standard answer.

\section{RESULT 1 AND DISCUSSION 6.1 Comparison of Paraphrases}

In this section, we summarize the experiments and results of using the different paraphrase based systems. We compare the performance of these systems, to two baselines:

- SurfSeeds: Uses only the surface-level seed patterns for extraction

- LexSynSeeds: Uses only the lexico-syntactic seed patterns for extraction

For the SurfPara, LexSynPara (Conv), and LexSynPara (Direct) systems, their performances were first measured using the top $25,50,75, \ldots, m$ patterns on the corresponding tuning sets. The configurations that performed the best on the tuning set were then applied to the test set. Figures 1, 2, and 3 summarize the results of the baselines and the paraphrase based systems for the terrorism, disease-outbreaks, and corporateacquisitions domains respectively using macro-averaged precision, recall, and f-scores.

\subsection{Discussion and Error Analysis}

Looking at the figures, it is clear that the SurfPara system does not improve much over the LexSynSeeds baseline (in fact sometimes it performs much worse). The LexSynPara (Conv) and LexSynPara (Direct) systems, however, consistently perform at par or improve over the baselines and the SurfPara system. This demonstrates the power of the generalization obtained by using the lexico-syntactic patterns. This generalization seems especially important in domain-specific IE: Limited redundancy in a small domain-specific corpus means that the system must have a large number of

\footnotetext{
${ }^{9}$ Template generation should logically follow the extraction step.
} 


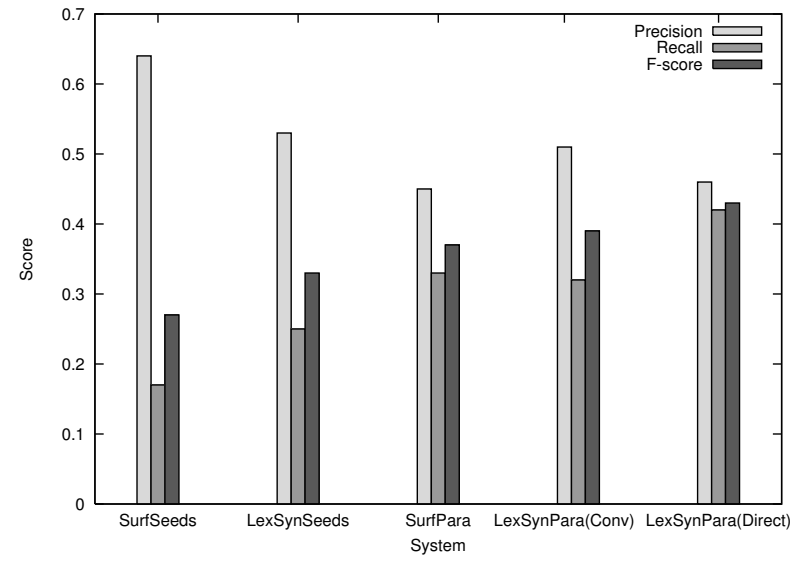

Figure 1: Paraphrase patterns in terrorism domain

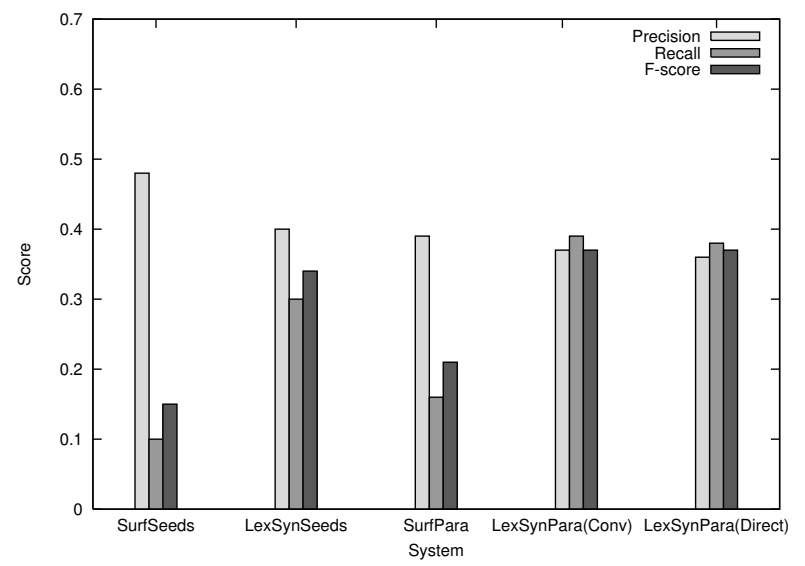

Figure 2: Paraphrase patterns in diseaseoutbreaks domain

patterns to achieve good recall ${ }^{10}$. Our analysis of the paraphrase patterns also confirms that generalization is crucial here $^{11}$. Also, the fact that LexSynPara (Direct) performs the best indicates that data sparseness is a problem in learning surface-level paraphrases. Analysis of the learned patterns also confirms this. We discuss the data sparseness problem in Section 7.2.

\section{RESULT 2 AND DISCUSSION}

\subsection{Comparison of Paraphrase Based and Traditional IE Systems}

In this section, we present experiments and results to compare the performance of our paraphrase patterns based IE system to some state-of-the-art patterns based IE systems trained using the traditional IE approach.

\footnotetext{
${ }^{10}$ Even for open-domain relation extraction, [3] point out that using surface-level patterns for extractions results in low recall.

${ }^{11}$ It should however be noted that automatic generalization sometimes produces very general patterns, due to parser error. Hence at times it harms precision.
}

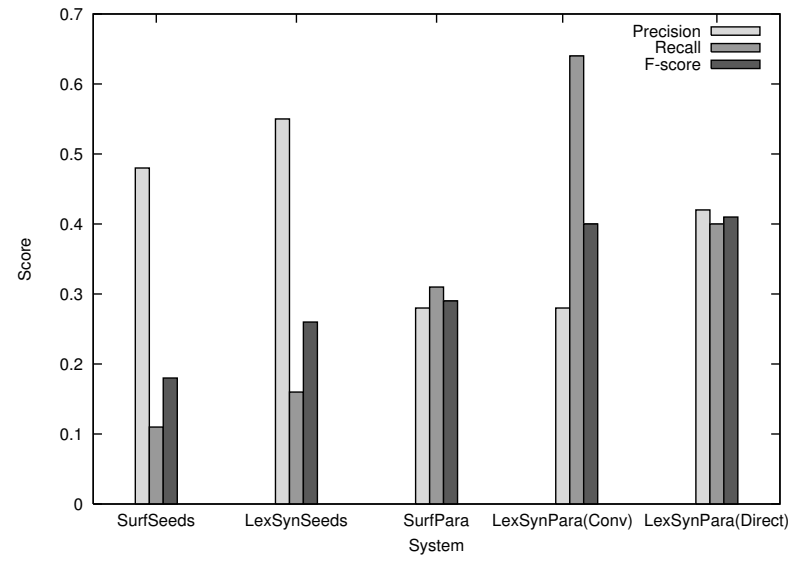

Figure 3: Paraphrase patterns in corporateacquisitions domain

For the terrorism and disease-outbreaks domain, we show results for the ASlog-TS [17] and the Semantic Affinity (SemAff) system [12]. Both these are weaklysupervised systems, that use a domain-specific corpus to learn extraction patterns. We also show the results of applying these systems in the relevant regions [12] - ASlog-TS (Rel) and SemAff (Rel). The performance numbers are obtained from [12]. These numbers are directly comparable to ours as we use the same test sets and evaluation methodology as them. For the corporate-acquisitions domain, we show the results of the SRV and SRVlng systems [8], both of which are supervised learning systems trained on domain-specific annotated data. The system scores are from [8]. These numbers are not directly comparable to ours, since we use a different evaluation methodology from theirs. These numbers are shown here only to give the readers a rough idea of performance in this domain. Figures 4,5 , and 6 summarize the results for the terrorism, disease-outbreaks, and corporate-acquisitions domains respectively using macro-averaged precision, recall, and f-scores.

\subsection{Discussion and Error Analysis}

From Figures 4, 5, and 6, it can be seen that overall, the performance of the best paraphrase patterns based system - LexSynPara (Direct), is comparable to the domain-specific IE systems.

While we are satisfied by the overall performance of the LexSynPara (Direct) system, we found that our approach performed badly on two slots - perpetrator individuals in the terrorism domain and victims in the disease-outbreaks domain. We investigated the cause for its poor performance on the these two slots. Intuition tells us, that it would be much easier to learn patterns for slots that extract entities whose event-roles are less ambiguous: Any context the entities occur in is likely to be a good disambiguator for them and hence 


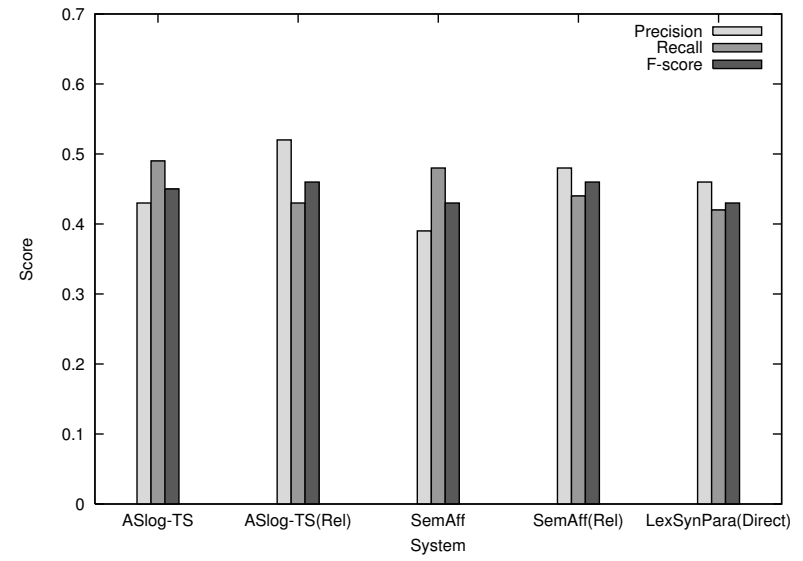

Figure 4: Paraphrase based vs traditional IE systems in terrorism domain

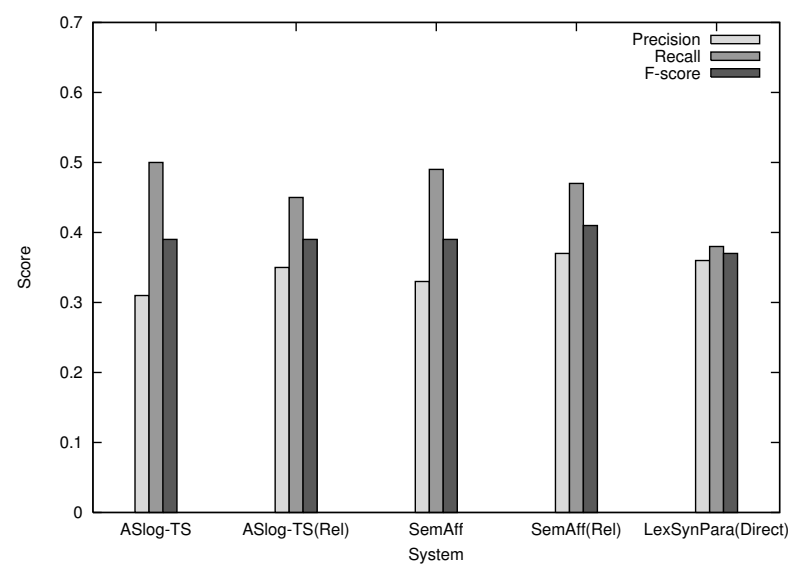

Figure 5: Paraphrase based vs traditional IE systems in disease-outbreaks domain

a good extraction-pattern. Another intuition tells us that it would be much easier to learn patterns for slots that extract entities belonging to smaller (in size) semantic classes, especially when using a broad-coverage corpus: The vectors for at least the high-frequency patterns corresponding to these slots would be much more dense compared to those of the patterns for slots that extract entities belonging to larger semantic classes.

The above two intuitions are confirmed by our analysis of the learned patterns and also explain our results. The slot - perpetrator individuals in the terrorism domain, extracts entities belonging to the general semantic class people, a class whose members can play many event roles both within and across domains (ex: members of the class people can also be victims, or onlookers or security personnel etc.) and is large in size. Looking at the learned patterns for this slot, we find that they also contain patterns for the slot victims. The second slot - victims in the disease-outbreaks domain, extracts entities that can belong to general semantic

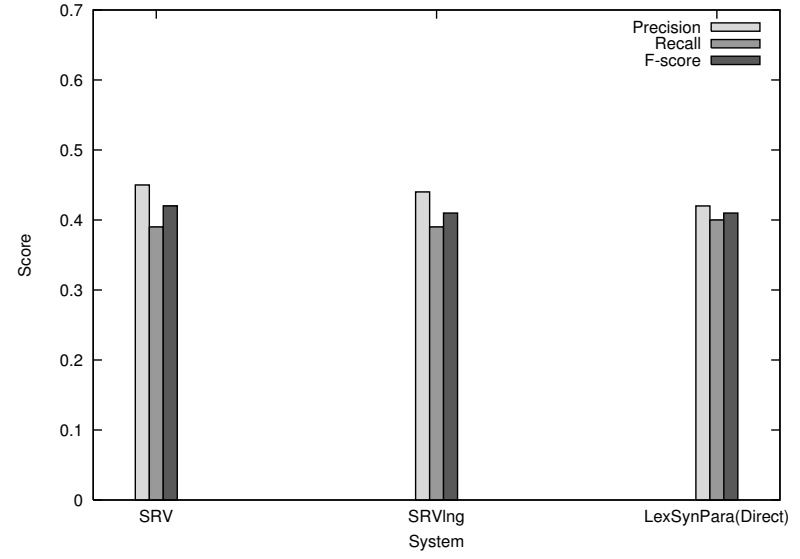

Figure 6: Paraphrase based vs traditional IE systems in corporate-acquisitions domain

classes people, animals, birds, etc., again classes whose members play different event-roles and which have large sizes. Our analysis of the patterns for this slot shows that some of the patterns are very general that extract animals, birds, etc. A third slot - victims in the terrorism domain, also suffers from the slot-filler ambiguity and sparse vector problems initially (when using SurfPara and LexSynPara (Conv) methods), but the problem becomes much less pronounced in the LexSynPara (Direct) method, where the vectors are less sparse. We hypothesize that if we were to use a corpus one order of magnitude larger than the one we have used and have the computation power to handle it, the paraphrases based method will perform much better. Unfortunately, we do not have access to such a corpus or the computation power to handle it currently. However, working with such a data set is feasible [3] and it is an avenue we want to explore. Finally, while it takes days to adapt any of the domain-specific methods to other domains, our method could be adapted to each of the new domains in a couple of hours.

\section{CONCLUSION}

Clearly, creating a knowledge-base containing paraphrases would be useful for various NLP applications. The paraphrase acquisition methods presented in this paper build paraphrase resources that are useful for information extraction. These paraphrase resources make IE for new event-roles and domains easy, and obviate the need to worry about the type of training data required. This observation leads to the conclusion that acquiring a knowledge-base containing paraphrases is an effective general methodology for information extraction. It also points to the need of building more such knowledge resources for other NLP applications.

\section{REFERENCES}

[1] M. Banko and O. Etzioni. The tradeoffs between traditional and open relation extraction. In 
Association for Computational Linguistics, pages 28-36, 2008.

[2] R. Barzilay, K. R. McKeown, and M. Elhadad. Information fusion in the context of multi-document summarization. In Association for Computational Linguistics, pages 550-557, 1999.

[3] R. Bhagat and D. Ravichandran. Large scale acquisiton of paraphrases for learning surface patterns. In Association for Computational Linguistics (ACL), 2008.

[4] E. Brill. Some advances in rule-based part of speech tagging. In Proceedings of the Twelfth National Conference on Artificial Intelligence, pages 722-727, Seattle, WA, 1994.

[5] C. Callison-Burch, P. Koehn, and M. Osborne. Improved statistical machine translation using paraphrases. In Human Language Technology Conference of the North American Chapter of the Association of Computational Linguistics, pages 17-24, 2006.

[6] M. S. Charikar. Similarity estimation techniques from rounding algorithms. In In Proceedings of the thiry-fourth annual ACM symposium on Theory of computing, pages 380-388, 2002.

[7] T. Cover and J. Thomas. Elements of Information Theory. John Wiley \& Sons, 1991.

[8] D. Freitag. Toward General-Purpose Learning for Information Extraction. In Proceedings of the 36th Annual Meeting of the Association for Computational Linguistics and 17th International Conference on Computational Linguistics, pages 404-408, Montreal, Quebec, August 1998.

[9] Z. Harris. Distributional structure. Word, pages 10(23):146-162, 1954.

[10] J. R. Hobbs, D. Appelt, J. Bear, D. Israel, M. Kameyalna, and M. Tyson. Fastus: A system for extracting information from text. In Proceedings of Human Language Technology Conference, 1993.

[11] D. Lin and P. Pantel. Dirt: Discovery of inference rules from text. In $A C M S I G K D D$ international conference on Knowledge discovery and data mining, pages 323-328, 2001.

[12] S. Patwardhan and E. Riloff. Effective Information Extraction with Semantic Affinity Patterns and Relevant Regions. In Proceedings of the 2007 Joint Conference on Empirical Methods in Natural Language Processing and Computational Natural Language Learning, pages 717-727, Prague, Czech Republic, June 2007.
[13] V. Pekar. Acquisition of verb entailment from text. In Proceedings of the Human Language Technology Conference of the NAACL, pages 49-56, New York City, USA, June 2006.

[14] D. Ravichandran and E. Hovy. Learning surface text for a question answering system. In Association for Computational Linguistics (ACL), Philadelphia, PA, 2002.

[15] D. Ravichandran, P. Pantel, and E. Hovy. Randomized algorithms and nlp: using locality sensitive hash function for high speed noun clustering. In In Proceedings of Association for Computational Linguistics, pages 622-629, 2005.

[16] E. Riloff. Automatically Constructing a Dictionary for Information Extraction Tasks. In Proceedings of the Eleventh National Conference on Artificial Intelligence, pages 811-816, Washington, DC, July 1993.

[17] E. Riloff. Automatically Generating Extraction Patterns from Untagged Text. In Proceedings of the Thirteenth National Conference on Articial Intelligence, pages 1044-1049, Portland, OR, August 1996.

[18] E. Riloff and W. Phillips. An Introduction to the Sundance and AutoSlog Systems. Technical Report UUCS-04-015, School of Computing, University of Utah, 2004.

[19] S. Sekine. On-demand information extraction. In In Proceedings of COLING/ACL, pages 731-738, 2006.

[20] Y. Shinyama, S. Sekine, and K. Sudo. Automatic paraphrase acquisition from news articles. In Proceedings of Human Language Technology Conference, pages 40-46, 2002.

[21] B. Sundheim. Overview of the Fourth Message Understanding Evaluation and Conference. In Proceedings of the Fourth Message Understanding Conference (MUC-4), pages 3-21, McLean, VA, June 1992.

[22] I. Szpektor and I. Dagan. Learning entailment rules for unary templates. In Proceedings of the International Conference on Computational Linguistics (COLING), pages 849-856, Manchester, UK, August 2008.

[23] I. Szpektor, H. Tanev, I. Dagan, and B. Coppola. Scaling web-based acquisition of entailment relations. In In Proceedings of Empirical Methods in Natural Language Processing, pages 41-48, 2004. 